\title{
A Novel Separated Position and Orientation System Integrated with Inertially Stabilized Platform
}

\author{
Yanshun Zhang $\mathbb{D}^{1},{ }^{1}$ Shuangji Feng $\mathbb{D}^{1},{ }^{1}$ Zhanqing Wang $\mathbb{D}^{2}{ }^{2}$ \\ Xiaopeng Xi $\oplus^{3},{ }^{3,4}$ and Ming Li $\oplus^{1}$ \\ ${ }^{1}$ School of Instrumentation and Optoelectronic Engineering, Beihang University, Beijing 100191, China \\ ${ }^{2}$ School of Automation, Beijing Institute of Technology, Beijing 100081, China \\ ${ }^{3}$ Tianjin Zhong Wei Aerospace Data System Technology Co., Ltd., Tianjin 300301, China \\ ${ }^{4}$ Tianjin Key Laboratory of Intelligent Information Processing in Remote Sensing, Tianjin 300100, China
}

Correspondence should be addressed to Shuangji Feng; qidaifsj6@buaa.edu.cn

Received 21 June 2017; Accepted 15 January 2018; Published 15 February 2018

Academic Editor: Sébastien Poncet

Copyright (c) 2018 Yanshun Zhang et al. This is an open access article distributed under the Creative Commons Attribution License, which permits unrestricted use, distribution, and reproduction in any medium, provided the original work is properly cited.

\begin{abstract}
Considering the application requirements of independent imaging payloads design, a novel scheme of separated position and orientation system (POS) is proposed, in which the high-precision inertial sensors of traditional centralized POS fixed on the imaging payloads are mounted on three gimbals of the inertially stabilized platform (ISP), respectively, and make them integrated. Then, the kinematics model of the ISP system is built to transmit the inertial information measured by separated inertial sensors mounted on ISP gimbals and flight body to the imaging payloads, calculating the position and attitude of the imaging payloads to achieve the function of separated POS. Based on the model, a series of simulations indicate that the precision difference between separated system and centralized system is ignorable under the condition of angular motion and variable velocity motion. Besides the effective function equal to traditional centralized system, the separated POS enhances the integration with the ISP. Moreover, it improves the design independence of the imaging payloads significantly.
\end{abstract}

\section{Introduction}

The remote sensing system is an effective way to achieve highresolution earth observations and obtain high-resolution images $[1,2]$. It has been widely applied in mapping, land resources exploration, disaster monitoring, and digital city construction [3-5]. Nowadays, common remote sensing system is composed of imaging payloads, position and orientation system (POS), and inertially stabilized platform (ISP). The imaging payloads are mounted on azimuth gimbal of the ISP system, and the POS consists of three gyros and three accelerometers are usually fixed on the imaging payloads so that the motion parameters of the imaging payloads can be measured by POS directly [6-8]. (It is called "centralized POS" below.)

In the centralized POS, extra angular rate gyros are mounted on roll, pitch, and azimuth gimbals of the ISP system, respectively, to achieve feed forward control [9-11], such as Swiss Leica PAV80 and PVA100, in which the centralized
POS is assembled inside the camera and additional piezoelectric gyros are located at three gimbals [12]. However, BUAA fixes the centralized POS on the shell of camera; meanwhile, the open-loop fiber optic gyros (or MEMS gyros) and quartz accelerometers are installed on each gimbal for rate feedback and initial leveling $[13,14]$. The traditional scheme in which imaging payloads, centralized POS and ISP, are designed independently and assembled separately requires the special installation space for POS to be reserved in or besides the imaging payloads, increasing the difficulty in designing imaging payloads independently [15]. Moreover, the gravity center of imaging payloads and POS supported by ISP changes when different types of centralized POS are adopted which introduces eccentric torque, and the torque is not easy to be eliminated by balance weight [16-18]. As a result, the control precision of the ISP system declines in dynamic situations.

Therefore, it is significant to explore the stabilization method of imaging payloads by integrating centralized POS 


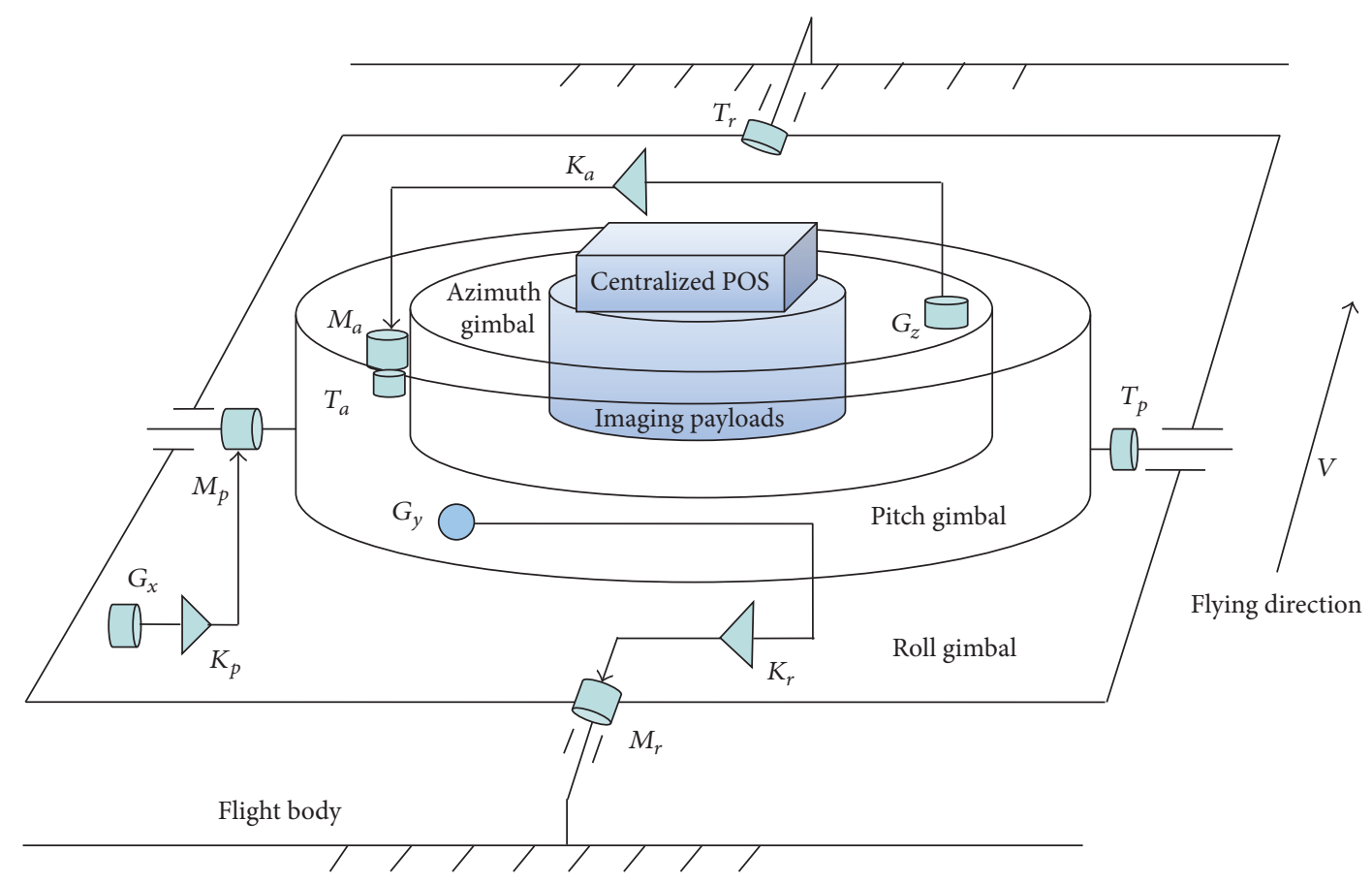

FIGURE 1: The composition of remote sensing system based on centralized POS.

with ISP system. It is technically feasible to realize the integrated design, in which the high-precision inertial sensors from centralized POS are mounted on three gimbals of the ISP. The design scheme proposed can simplify the mechanical interfaces among imaging payloads, POS and ISP. Besides, it improves the attitude stabilization accuracy of payloads effectively. A series of theoretical derivations and semiphysical simulations validate the feasibility of the scheme presented and the equivalence to performance of traditional centralized POS. Furthermore, the integrated design idea improves the design independence of the imaging payloads significantly.

\section{The Principle of Imaging Payloads Stabilization in Remote Sensing System}

Nowadays, the usual assembly mode of remote sensing system applied is that imaging payloads, centralized POS and ISP system, are designed independently and assembled separately [13]. The composition of remote sensing system based on centralized POS is shown in Figure 1.

From Figure $1, M_{i}(i=r, p, a)$ denote torque motors. Amplification gain coefficients are described by $K_{i}(i=$ $r, p, a) . T_{i}(i=r, p, a)$ represent high-precision angle sensors and $\dot{\theta}_{i}(i=r, p, a)$ are relative rotation angular velocities between gimbals. Three gyros and three accelerometers are assembled in the centralized POS to measure the attitude of imaging payloads fixed on the azimuth gimbal directly and provide reference for generating angular velocity command. Meanwhile, low-cost and small-sized gyros $G_{i}(i=x, y, z)$ are mounted, respectively, on three gimbals of the ISP system to provide the corresponding angular velocity information and conduct feedback control $[19,20]$.

When the system works normally, the position, velocity, and attitude information of imaging payloads provided by centralized POS can be used to generate angular velocity command which transformed into torque motor control signals, driving three gimbals to expected position. However, since the centralized POS is fixedly connected with the imaging payloads, it is necessary to reserve special installation space for POS near the imaging payloads, which increases the difficulty in designing imaging payloads independently. In addition, applying different types of POS may lead to the change of the gravity center of imaging payloads, which has an impact on the control precision of the ISP system. Furthermore, the convenience of mechanical interfaces between the imaging payloads and the ISP system declines.

\section{The Design Scheme of Separated POS}

Most of the imaging payloads in large remote sensing system are special equipment and they need to be designed independently. Therefore, it is significant to integrate attitude measurement components with the ISP system. This paper presents the separated POS design idea, in which the inertial sensors from original centralized POS are mounted on three gimbals of the ISP system separately to make POS and ISP integrated. Then, the kinematics model of the ISP system is built to transmit the inertial information from each gimbal and flight inertial navigation system to the azimuth gimbal. The advantages of the design scheme proposed in this paper are that the separated POS keeps the equivalent 
performance with traditional centralized POS, but it reduces the number of inertial devices and simplifies the mechanical and electronic interfaces between the imaging payloads and ISP system compared with traditional large remote sensing system. Moreover, the separated system enhances the control accuracy of the ISP system and the design independence of the imaging payloads significantly.

3.1. Coordinates Commonly Used in Centralized POS and ISP System. Imaging payloads usually need to be stable in local geographical coordinate. One motion vector may have different expressions in different coordinates. Therefore, the motion vectors should be decomposed to the specified coordinates in attitude determination and gimbals control. The coordinates used normally in inertially stabilized platform are shown as follows:

(1) Navigation coordinate $O x_{n} y_{n} z_{n}: x_{n}, y_{n}$, and $z_{n}$ denote the east, north, and up directions of the local horizon.

(2) Base coordinate $O x_{b} y_{b} z_{b}: x_{b}, y_{b}$, and $z_{b}$ represent the right, forward, and up directions of the flight body.

(3) Roll coordinate $O x_{r} y_{r} z_{r}$ : roll axes $y_{r}$ and $y_{b}$ are in the same direction, and the roll coordinate is rotated roll angle $\theta_{r}$ around axis $y_{b}$ with respect to base coordinate.

(4) Pitch coordinate $O x_{p} y_{p} z_{p}$ : pitch axis $x_{p}$ is coaxial with $x_{r}$, and the pitch coordinate is turned pitch angle $\theta_{p}$ around axis $x_{r}$ with respect to roll coordinate.

(5) Azimuth coordinate $O x_{a} y_{a} z_{a}$ : azimuth axis $z_{a}$ is collinear with $z_{p}$, and the azimuth coordinate is rotated azimuth angle $\theta_{a}$ around axis $z_{p}$ with respect to pitch coordinate.

Roll angle $\theta_{r}$, pitch angle $\theta_{p}$, and azimuth angle $\theta_{a}$ stand for the rotation angles of roll-pitch-azimuth coordinates with respect to body coordinate [6]. (The positive rotation is counterclockwise.) The variables in different coordinates can be converted mutually through these angles. The coordinates of the ISP system and the conversion relationship are shown in Figure 2.

3.2. The Design Philosophy of Separated POS. The key points of separated POS integrated with ISP system are as follows: the inertial sensors (three gyros and three accelerometers) are no longer fixedly connected with imaging payloads as centralized POS; they are separately mounted on three gimbals of the ISP system to make up separate inertial measurement units. According to the kinematics model of the ISP system built below, the motion information provided by inertial sensors fixed on three gimbals and the information provided by flight inertial navigation system are transmitted to imaging payloads, generating the inertial measurement signals which reflect the motion status of imaging payloads. Utilizing the signals with inertial navigation algorithm can achieve the performance of separated POS. The scheme is equivalent to fixing virtual centralized POS on the imaging payloads, which is called "virtual centralized POS" in Figure 3. The composition of remote sensing system with separated POS is shown in Figure 3.

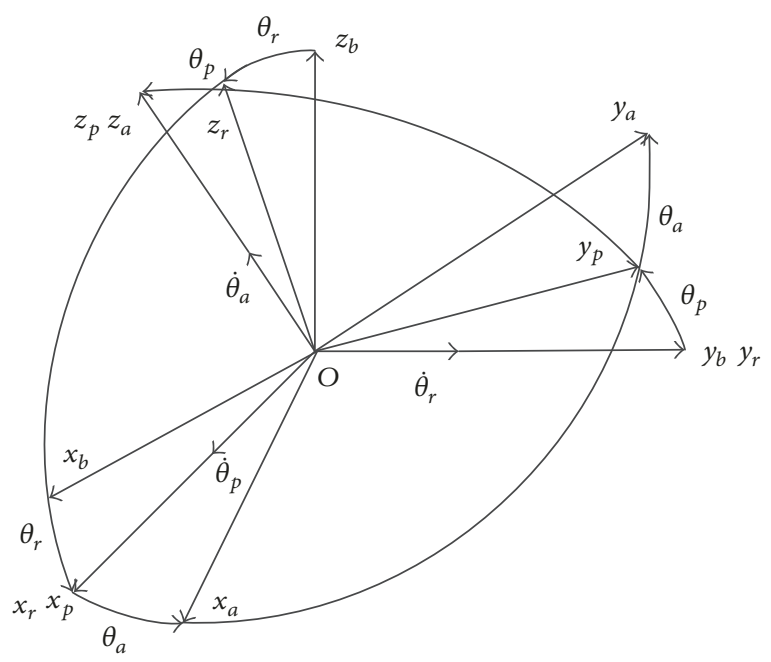

Figure 2: Gimbals coordinate converted diagrammatic sketch.

In Figure 3, the sensitive axes of three gyros are, respectively, coaxially mounted with roll axis, pitch axis, and azimuth axis of gimbals to measure angular velocities of three frames with respect to inertial space. Two accelerometers $A_{x}, A_{y}$ are orthogonally mounted on the pitch gimbal and their sensitive axes are, respectively, collinear with $x_{p}$ and $y_{p}$ to measure specific forces of the pitch gimbal in horizontal direction. Another accelerometer $A_{z}$ is fixed on the azimuth gimbal and its sensitive axis is collinear with $z_{a}$ of azimuth coordinate to measure specific forces of payloads along $z$-axis directly. Angle sensors are installed at the rotation axis of each gimbal and their sensitive axes are coaxial with each gimbal spin axis to measure the relative angles between gimbals. The flight inertial device is fixed on flight body to measure inertial information $\omega_{i b}$.

In this scheme, the palstances $\omega_{i r}, \omega_{i p}, \omega_{i a}$ of gimbals with respect to inertial space and angular velocity $\omega_{i b}$ of flight body constitute the angular velocity of azimuth coordinate attached to imaging payloads. Therefore, the outputs of gyros and flight inertial navigation system constitute the angular velocities equal to the measurements provided by centralized POS, namely, achieving the performance of separated POS.

Compared with centralized POS, the separated POS adopted in remote sensing system has the following advantages: firstly, the integrated design scheme simplifies the mechanical interfaces between imaging payloads and POS/ ISP as well as improving the design independence. Secondly, the gyros mounted on three gimbals can also provide rate feedback information, which enhances the feedback control precision and improves the synchronization between motion measurement and control command significantly. Thirdly, the number of inertial sensors declines in contrast to traditional centralized POS.

3.3. The Kinematics Model of the Separated POS Integrated with ISP System. Since the inertial sensors of the separated POS are mounted on three gimbals of the ISP system, the angular velocities and specific forces output cannot reflect the motion status of the imaging payloads fixed on the azimuth 


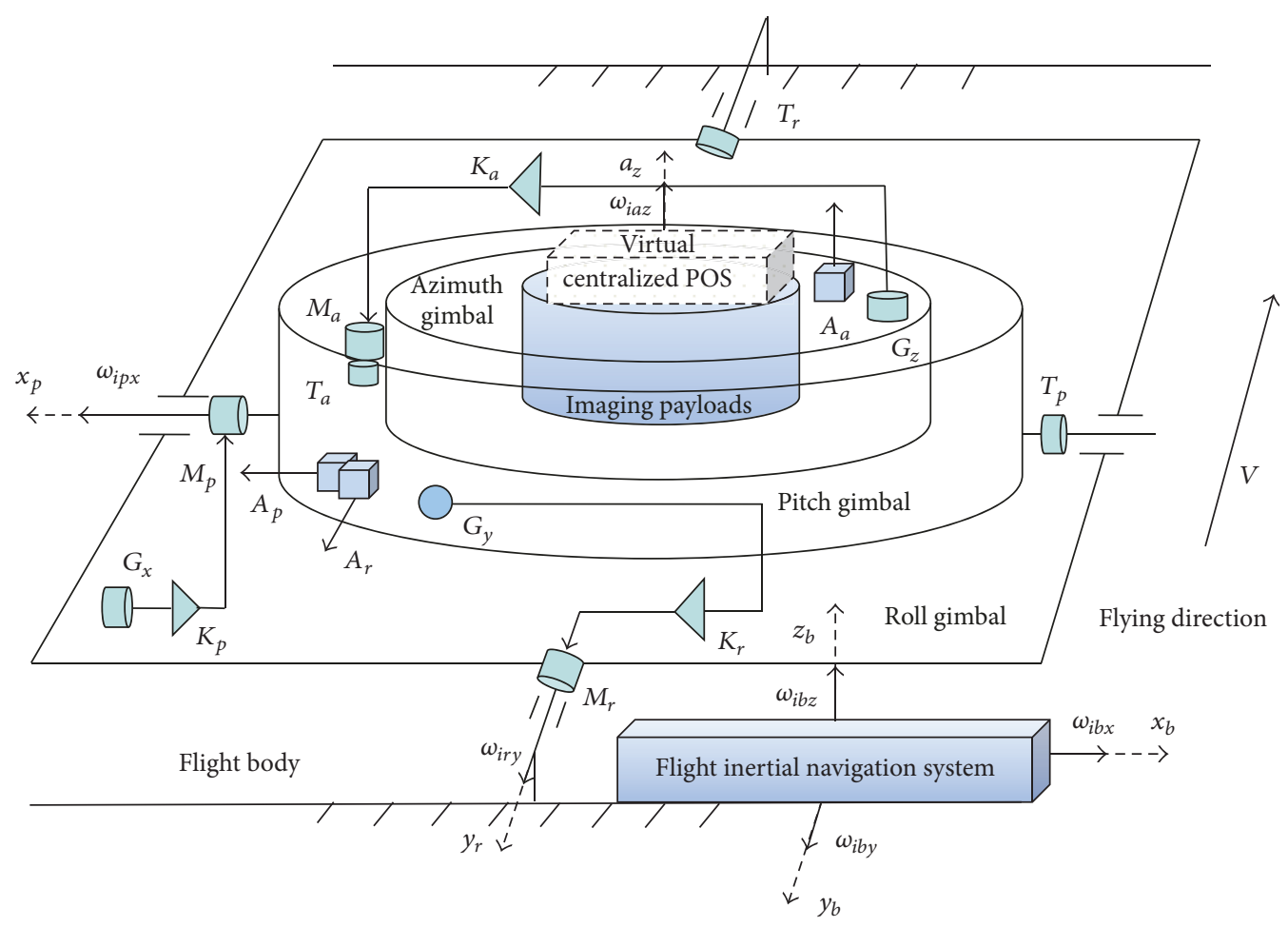

FIGURE 3: Diagram of remote sensing system with separated POS.

gimbal directly. Therefore, it is necessary to convert and transmit the signal from the flight inertial navigation system and the inertial sensors of separated POS to the azimuth gimbal and then obtain the motion information of imaging payloads and realize the attitudes measurement and stability. In other words, virtual centralized POS is generated correspondingly by converting the information from inertial sensors of the separated POS to imaging payloads. The main content of this section consists of the conversion and transmission of inertial signals from gyros and accelerometers.

\subsubsection{The Conversion and Transmission of Measurement Signal} from Gyros. The angular velocities of three gimbals with respect to inertial space are measured by gyros fixed on gimbals. The gyros in flight navigation system can provide the angular velocity of flight body with respect to inertial space. They need to be converted and transmitted to imaging payloads to calculate attitudes and generate control command. As follows, the conversion and transmission model of inertial angular velocities is derived.

Assume that $\vec{\omega}_{i b}^{b}=\left[\begin{array}{lll}\omega_{i b x} & \omega_{i b y} & \omega_{i b z}\end{array}\right]^{T}$ denotes angular velocity of flight body. The angular velocity of roll gimbal with respect to inertial space is expressed as $\vec{\omega}_{i r}^{r}=$ $\left[\begin{array}{lll}\omega_{i r x} & \omega_{i r y} & \omega_{i r z}\end{array}\right]^{T} \cdot \vec{\omega}_{i p}^{p}=\left[\begin{array}{lll}\omega_{i p x} & \omega_{i p y} & \omega_{i p z}\end{array}\right]^{T}$ represents the angular velocity of pitch gimbal with relative to inertial space. $\vec{\omega}_{i a}^{a}=\left[\begin{array}{lll}\omega_{i a x} & \omega_{i a y} & \omega_{i a z}\end{array}\right]^{T}$ is the angular velocity of azimuth gimbal with relative to inertial space. And the angular velocities of the gimbals obtained by pitch-roll-azimuth sequence of rotations are described by $\dot{\theta}_{p}, \dot{\theta}_{r}$, and $\dot{\theta}_{a}$, respectively.

Firstly, the transmission relationship of angular velocities between flight body and roll gimbal is discussed. Since the roll gimbal is mounted on the base of flight body, the angular velocity of roll gimbal is comprised of angular velocity of flight body and spin velocity of roll gimbal. The angular velocity of flight body is projected to roll gimbal and added to spin velocity of roll gimbal; the result is given by

$$
\vec{\omega}_{i r}^{r}=C_{b}^{r} \vec{\omega}_{i b}+\vec{\omega}_{b r}=\left[\begin{array}{c}
\omega_{i b x} \cos \theta_{r}-\omega_{i b z} \sin \theta_{r} \\
\omega_{i b y}+\dot{\theta}_{r} \\
\omega_{i b x} \sin \theta_{r}+\omega_{i b z} \cos \theta_{r}
\end{array}\right] .
$$

Secondly, on the base of angular velocity of roll gimbal obtained, the transmission rule of angular velocities between roll and pitch gimbals is discussed. Since the rotation axis of pitch gimbal is fixed on the roll gimbal and orthogonal to the rotation axis of roll gimbal, the angular velocity of pitch frame is made up of angular velocity of roll gimbal and spin velocity of pitch gimbal. The velocity of roll gimbal is projected to pitch gimbal and added to spin velocity of pitch gimbal; the result is given by

$$
\vec{\omega}_{i p}^{p}=C_{r}^{p} \vec{\omega}_{i r}+\vec{\omega}_{r p}=\left[\begin{array}{c}
\omega_{i r x}+\dot{\theta}_{p} \\
\omega_{i r y} \cos \theta_{p}+\omega_{i r z} \sin \theta_{p} \\
-\omega_{i r y} \sin \theta_{p}+\omega_{i r z} \cos \theta_{p}
\end{array}\right] .
$$


Thirdly, the angular velocity of azimuth gimbal is obtained from projection of angular velocity of pitch gimbal. Since the azimuth gimbal is installed in the pitch gimbal through bearings, the rotation axis of azimuth gimbal is coaxial with the axis $z_{a}$ of azimuth coordinate. Considering the spin velocity of azimuth gimbal, the angular velocity of azimuth gimbal can be expressed as

$$
\vec{\omega}_{i a}^{a}=C_{p}^{a} \vec{\omega}_{i p}^{p}+\vec{\omega}_{p a}=\left[\begin{array}{c}
\omega_{i p x} \cos \theta_{a}+\omega_{i p y} \sin \theta_{a} \\
-\omega_{i p x} \sin \theta_{a}+\omega_{i p y} \cos \theta_{a} \\
\omega_{i p z}+\dot{\theta}_{a}
\end{array}\right] .
$$

According to (1), (2), and (3), the angular velocity of imaging payloads with respect to inertial space is given by

$$
\begin{aligned}
& \vec{\omega}_{i a}^{a}=\left[\begin{array}{c}
\omega_{i a x} \\
\omega_{i a y} \\
\omega_{i a z}
\end{array}\right] \\
& =\left[\begin{array}{ccc}
\cos \theta_{a} \cos \theta_{r}+\sin \theta_{a} \sin \theta_{p} \sin \theta_{r} & \sin \theta_{a} \cos \theta_{p} & \sin \theta_{a} \sin \theta_{p} \cos \theta_{r}-\cos \theta_{a} \sin \theta_{r} \\
-\cos \theta_{r} \sin \theta_{a}+\cos \theta_{a} \sin \theta_{p} \sin \theta_{r} & \cos \theta_{a} \sin \theta_{p} & \cos \theta_{a} \sin \theta_{p} \cos \theta_{r}+\sin \theta_{a} \sin \theta_{r} \\
\cos \theta_{p} \sin \theta_{r} & -\sin \theta_{p} & \cos \theta_{p} \cos \theta_{r}
\end{array}\right]\left[\begin{array}{c}
\omega_{i b x} \\
\omega_{i b y} \\
\omega_{i b z}
\end{array}\right] \\
& +\left[\begin{array}{ccc}
\cos \theta_{p} \sin \theta_{a} & \cos \theta_{a} & 0 \\
\cos \theta_{a} \cos \theta_{p} & -\sin \theta_{p} & 0 \\
-\sin \theta_{p} & 0 & 1
\end{array}\right]\left[\begin{array}{c}
\dot{\theta}_{r} \\
\dot{\theta}_{p} \\
\dot{\theta}_{a}
\end{array}\right]
\end{aligned}
$$

From (4), the angular velocity of imaging payloads with respect to inertial space can be obtained according to angular velocities of flight body $\omega_{i b x}, \omega_{i b y}$, and $\omega_{i b z}$ and spin velocities of gimbals $\dot{\theta}_{r}, \dot{\theta}_{p}$, and $\dot{\theta}_{a}$; then angular velocity command can be generated to make platform stable. However, the precision of rotational rates between gimbals is limited by angle sensors mounted along rotation axis of gimbals. The angular velocities are calculated by angle differential, but the deviation of angle differential is so large that the stabilization precision of imaging payloads declines. Therefore, it is significant to enhance the stabilization of platform by improving the measurement precision of spin velocities of gimbals.

The precision of angular velocity measured by gyro is much higher than angle differential of angle sensor. Therefore, it can improve the stabilization of platform by fixing gyros on three gimbals to measure spin velocities of gimbals.

$$
\begin{gathered}
\omega_{i r y}=\omega_{i b y}+\dot{\theta}_{r} \\
\omega_{i p x}=\omega_{i r x}+\dot{\theta}_{p} \\
\omega_{i a z}=\omega_{i p z}+\dot{\theta}_{a} .
\end{gathered}
$$

Thus, the angular velocities of gimbals with respect to inertial space can be measured directly by gyros instead of output derivation from angle sensors.

According to (1) and (2), it can be known that

$$
\begin{gathered}
\omega_{i p x}^{p}=\omega_{i r y}^{r} \cos \theta_{p}+\omega_{i r z}^{r} \sin \theta_{p} . \\
\omega_{i r z}^{r}=\omega_{i b x}^{b} \sin \theta_{r}+\omega_{i b z}^{b} \cos \theta_{r}
\end{gathered}
$$

Substituting (7) into (6), one obtains

$$
\omega_{i p y}^{p}=\omega_{i r y}^{r} \cos \theta_{p}+\left(\omega_{i b x}^{b} \sin \theta_{r}+\omega_{i b z}^{b} \cos \theta_{r}\right) \sin \theta_{p} .
$$

Substituting (8) into (3), one obtains

$$
\begin{gathered}
{\left[\begin{array}{c}
\omega_{\text {iax }} \\
\omega_{\text {iay }} \\
\omega_{\text {iaz }}
\end{array}\right]=\left[\begin{array}{ccc}
\cos \theta_{a} & \cos \theta_{p} \sin \theta_{a} & 0 \\
-\sin \theta_{a} & \cos \theta_{p} \cos \theta_{a} & 0 \\
0 & 0 & 1
\end{array}\right]\left[\begin{array}{c}
\omega_{i p x} \\
\omega_{i r y} \\
\omega_{i a z}
\end{array}\right]} \\
+\left[\begin{array}{ccc}
\sin \theta_{r} \sin \theta_{p} \sin \theta_{a} & 0 & \cos \theta_{r} \sin \theta_{p} \sin \theta_{a} \\
\sin \theta_{r} \sin \theta_{p} \cos \theta_{a} & 0 & \cos \theta_{r} \sin \theta_{p} \cos \theta_{a} \\
0 & 0 & 0
\end{array}\right]\left[\begin{array}{l}
\omega_{i b x} \\
\omega_{i b y} \\
\omega_{i b z}
\end{array}\right] .
\end{gathered}
$$

From (9), it can be seen that angular velocities of imaging payloads with respect to inertial space can be obtained according to the angular velocities of gimbals $\omega_{i p x}, \omega_{i r y}$, and $\omega_{i a z}$ and the angular velocity of flight body $\omega_{i b}$.

3.3.2. The Conversion and Transmission of Measurement Signal from Accelerometers. The sensitive axes of three accelerometers are always orthogonal, which decreases the difficulty in decoupling. According to the principle of coordinate transformation, the outputs of two accelerometers fixed on the pitch gimbal are projected to azimuth coordinate to get three-axis acceleration of azimuth gimbal

$$
\left[\begin{array}{l}
\operatorname{acc}_{a x} \\
\operatorname{acc}_{a y} \\
\operatorname{acc}_{a z}
\end{array}\right]=\left[\begin{array}{ccc}
\cos \theta_{a} & \sin \theta_{a} & 0 \\
-\sin \theta_{a} & \cos \theta_{a} & 0 \\
0 & 0 & 1
\end{array}\right]\left[\begin{array}{l}
\mathrm{acc}_{p x} \\
\operatorname{acc}_{p y} \\
\operatorname{acc}_{a z}
\end{array}\right] .
$$

From (10), the information of the accelerometers fixed separately on different gimbals is utilized to obtain the output of accelerometers equal to that in centralized POS.

Therefore, the inertial information measured by gyros and accelerometers fixed on gimbals and the signals generated from flight inertial navigation system can be transmitted 
to the imaging payloads according to (9) and (10). After attitude calculation, the corresponding function of separated POS can be realized.

\section{Simulation}

To validate the feasibility and precision of the separated POS proposed in this paper, the semiphysical simulations are conducted. The scheme is as follows:

(1) Utilize trajectory generator to produce exact navigation data (including the output of three gyros and three accelerometers, attitude, and position information of the imaging payloads) in different motion conditions to test the performance of the separated POS and the centralized POS.

(2) Add the sensor errors which reflect the sensor performance to the exact data output by trajectory generator to generate semiphysical simulation data.

(3) Read the semiphysical data to calculate the attitude of imaging payloads in the separated POS and the centralized POS, respectively.

(4) Compare the calculation results with the exact attitude from trajectory generator to test the equivalence of the main performance between the separated POS and the centralized POS.

Two kinds of experiments are designed under angular motion and variable rectilinear motion to compare the performances of the separated POS and the centralized POS.

4.1. The Performance Comparison of Separated POS and Centralized POS in Angular Motion. When angular motion of gimbals exists, the outputs of gyros and accelerometers fixed on gimbals are projected to the azimuth gimbal through angles provided by angle sensors. Thus, the precision of the angle sensors has an effect on the performance of separated POS. This section analyzes the performance of separated POS and centralized POS when the angle sensor error exists or not.

4.1.1. Trajectory Generation. The ideal navigation data of the separated POS and the centralized POS is generated, respectively, by trajectory generator, and the length of data is $600 \mathrm{~s}$. In the process, the base of platform keeps motionless and the three gimbals keep rotating around each axis at the uniform speeds. The azimuth angle ranges from $0^{\circ}$ to $20^{\circ}$, the pitch angle ranges from $0^{\circ}$ to $10^{\circ}$, and the roll angle ranges from $0^{\circ}$ to $5^{\circ}$. The attitude variation curve of the platform is shown in Figure 4.

Based on the ideal data generated by trajectory generator, the sensor errors reflecting real sensor performance are added to produce semiphysical simulation data. The constant drifting of gyro is $0.01^{\circ} / \mathrm{h}$ and the constant bias of accelerometer is $50 \mathrm{ug}\left(\mathrm{g}=9.7803267714 \mathrm{~m} / \mathrm{s}^{2}\right)$.

4.1.2. The POS Performance Comparison Experiments without Angle Sensor Error. After reading the semiphysical simulation data added to inertial sensor errors from gyro and
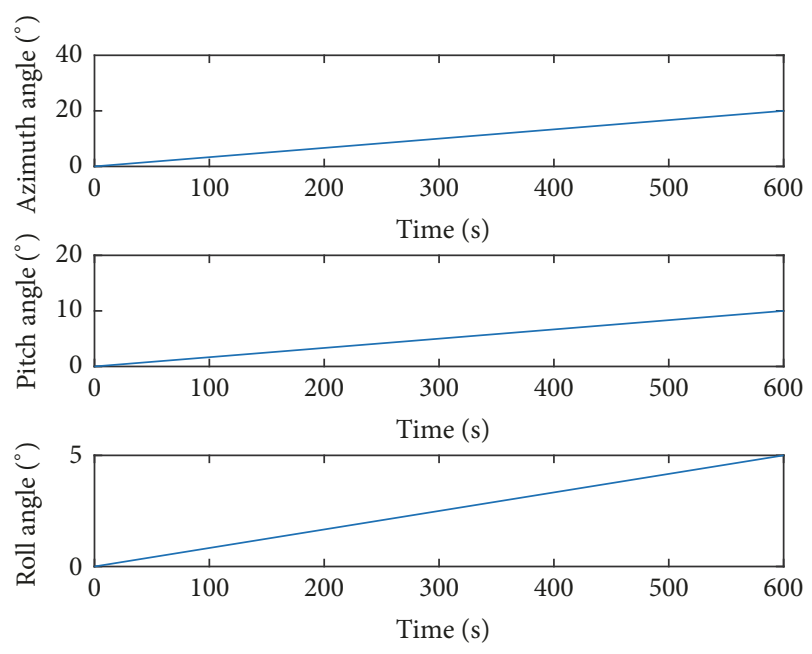

FIGURE 4: The platform attitude generated by trajectory generator.

accelerometer, the attitude of the imaging payloads is calculated by the separated POS and the centralized POS, respectively. Then, the comparison between calculation results and ideal attitude from trajectory generator is carried out. The attitude errors are shown in Figure 5(a); the differences between attitude errors of separated POS and centralized POS are shown in Figure 5(b). According to Figure 5, the attitude error characteristics can be displayed visually to analyze whether the performance of separated POS is equal to centralized POS or not when angle sensor error does not exist.

From Figure 5, the attitude errors of separated POS and centralized POS are similar. And the maximum three-axis attitude (azimuth angle, pitch angle, and roll angle) errors of separated POS/centralized POS, caused by errors from gyro and accelerometer, are $7.653 \times 10^{-4^{\circ}} / 7.593 \times 10^{-4^{\circ}}$, $2.151 \times 10^{-3^{\circ}} / 2.148 \times 10^{-3^{\circ}}$, and $1.037 \times 10^{-3^{\circ}} / 1.037 \times$ $10^{-3^{\circ}}$, respectively. The minute differences of attitude errors between separated POS and centralized POS during the whole process are shown in Figure 5(b). The maximum differences of the three-axis attitude errors are $7.353 \times 10^{-7^{\circ}}$, $2.978 \times 10^{-6^{\circ}}$, and $5.772 \times 10^{-6^{\circ}}$, respectively, which can be ignored compared with the corresponding attitude errors shown in Figure 5(a). Therefore, the equivalence of the separated POS and the centralized POS is validated when angle sensor error does not exist.

4.1.3. The POS Performance Comparison Experiments with Angle Sensor Error. The angle sensor error $2^{\prime \prime}$ is added to inertial data generated by trajectory generator. And the attitudes calculated by separated POS and centralized POS, respectively, are compared to ideal attitudes. The attitude errors are shown in Figure 6(a), and the minute differences between the attitude errors of two POSs are shown in Figure 6(b). Based on Figure 6, the attitude error characteristics can be displayed visually to analyze whether the performance of separated POS is equal to centralized POS or not when angle sensor error exists. 

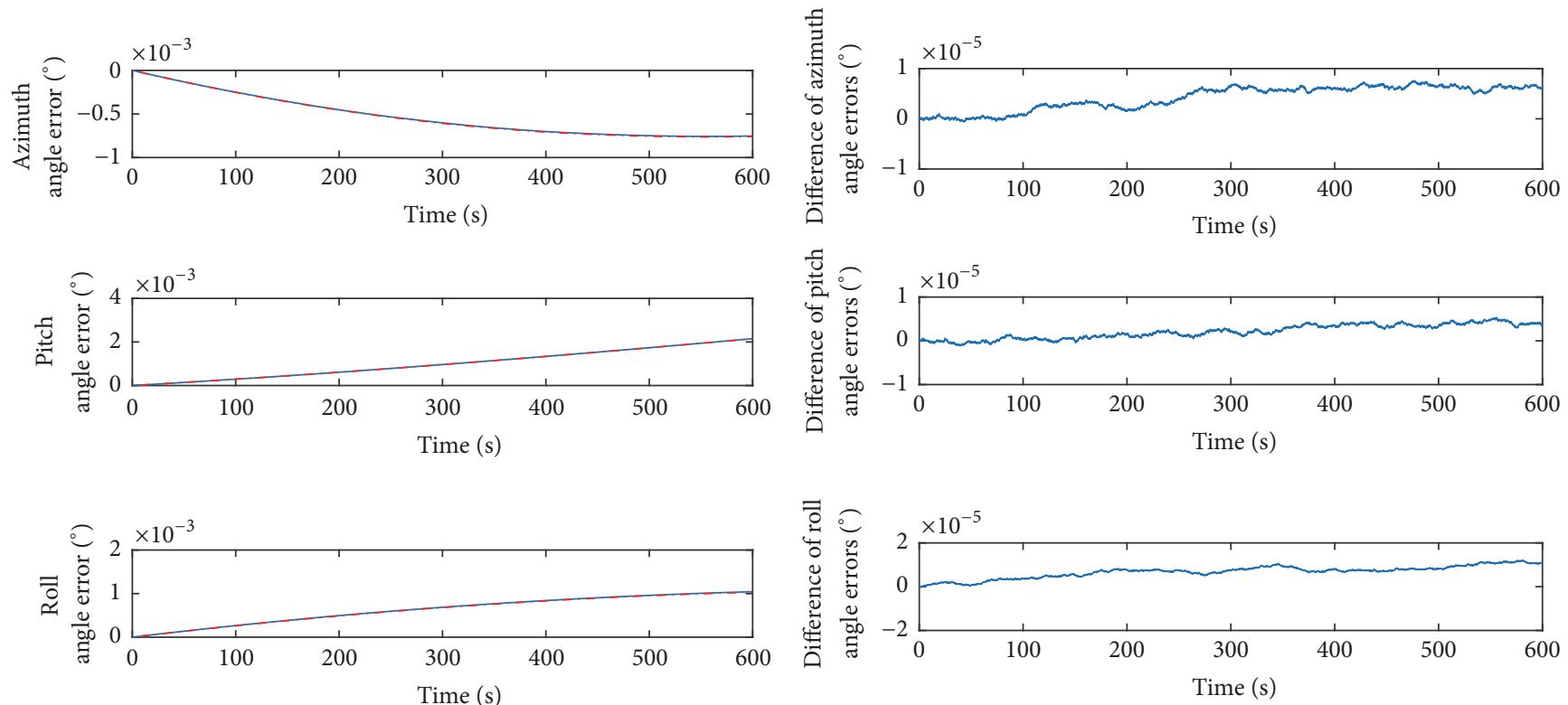

— Separated pos attitude error

...- Centralized pos attitude error

(a) The attitude errors of separated POS and centralized POS compared with ideal attitudes

(b) The differences between attitude errors of separated POS and centralized POS

FIGURE 5: The attitude errors without angle sensor error.
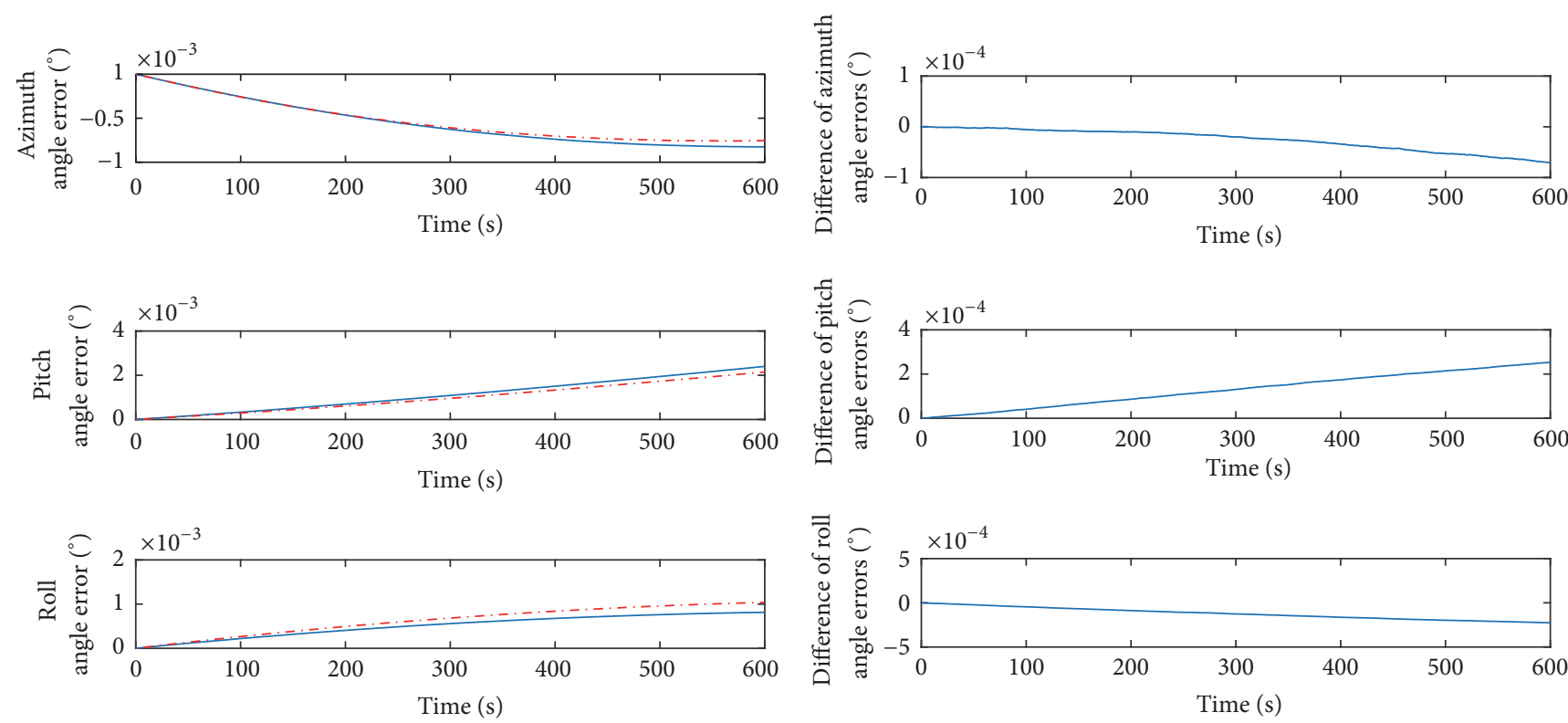

- Separated POS attitude error

-.. Centralized POS attitude error

(a) The attitude errors of separated POS and centralized POS compared with the ideal attitudes

(b) The differences between attitude errors of separated POS and centralized POS

FIGURE 6: The attitude errors with angle sensor error. 

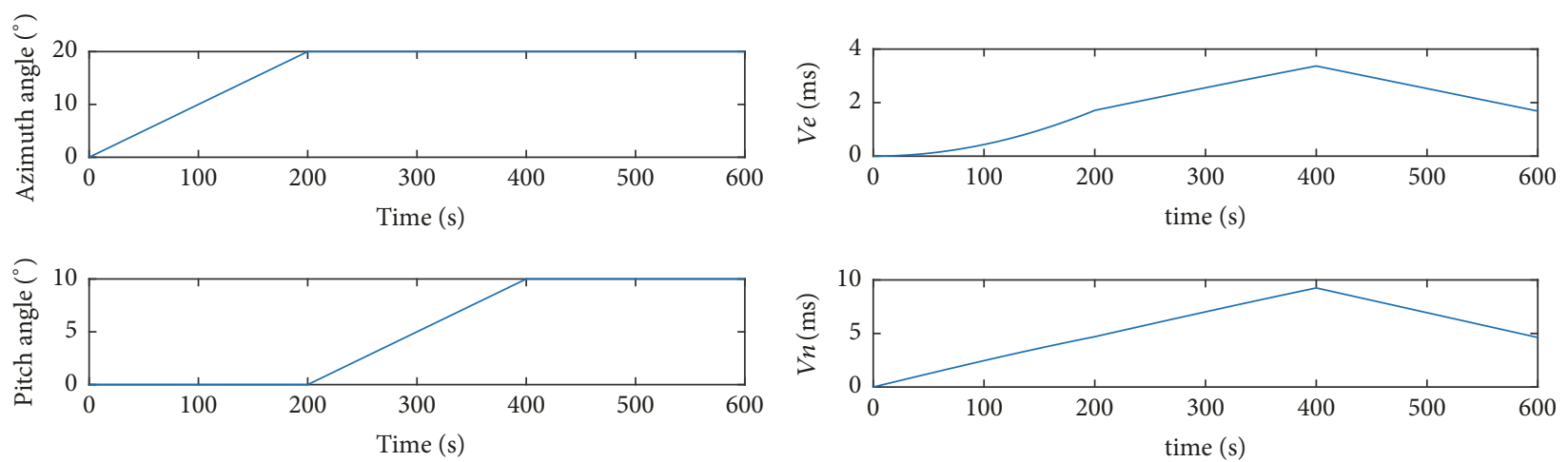

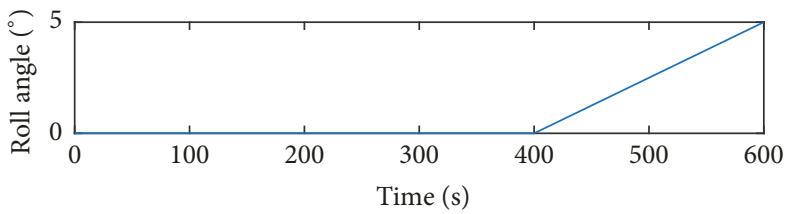

(a) The attitude angle of platform

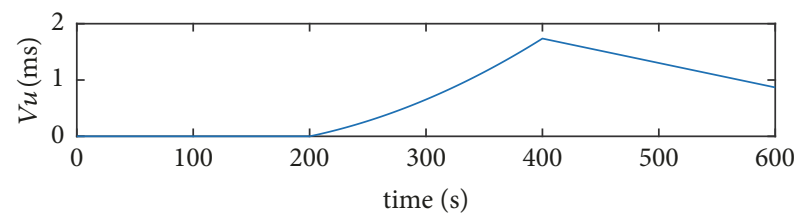

(b) The linear velocity of flight body

FIGURE 7: The attitude angle of platform and linear velocity of flight body produced by trajectory generator.

From Figure 6, the attitude errors of separated POS and centralized POS are similar. And the maximum three-axis attitude (azimuth angle, pitch angle, and roll angle) errors of separated POS/centralized POS, caused by errors added from gyro, accelerometer, and angle sensor, are $8.331 \times$ $10^{-4^{\circ}} / 7.593 \times 10^{-4^{\circ}}, 2.399 \times 10^{-3^{\circ}} / 2.148 \times 10^{-3^{\circ}}$, and $8.057 \times$ $10^{-4^{\circ}} / 1.033 \times 10^{-3^{\circ}}$, respectively. The small differences between attitude errors of separated POS and centralized POS during the whole process are shown in Figure 6(b). The maximum differences of the three-axis attitude errors are $7.383 \times$ $10^{-5^{\circ}}, 2.513 \times 10^{-4^{\circ}}$, and $2.271 \times 10^{-4^{\circ}}$, respectively, which can be ignored compared with the corresponding attitude errors shown in Figure 6(a).

Comparing the three-axis attitude errors in Figures 5(b) and 6(b), the differences between the attitude errors of two POSs increase after adding the angle sensor error, and the corresponding maximum values are $6.747 \times 10^{-5^{\circ}}, 2.483 \times$ $10^{-4^{\circ}}$, and $2.213 \times 10^{-4^{\circ}}$, respectively. They can also be neglected in contrast to the attitude errors calculated by separated POS and centralized POS, respectively. Therefore, the equivalence of the separated and centralized POS is not influenced by angle sensor error.

4.2. The Performance Comparison of Separated POS and Centralized POS in Variable Rectilinear Motion. In the actual flight process, the variable rectilinear motion of flight body has an effect on attitude calculation precision of the inertially stabilized platform. Thus, the influence of linear acceleration from flight body is analyzed in the separated POS and the centralized POS. Besides, the performance comparison of two POSs is also discussed.

4.2.1. Trajectory Generation. The ideal navigation data of the separated POS and the centralized POS is, respectively, generated by trajectory generator, and the length of data is $600 \mathrm{~s}$. In the process, the velocity of flight body and the attitude of imaging payloads change synchronously. The trajectory includes three parts (T1, T2, and T3) and each part sustains $200 \mathrm{~s}$. In part $T 1$, the azimuth angle ranges from $0^{\circ}$ to $20^{\circ}$ and the velocity of flight body changes from 0 to $5 \mathrm{~m} / \mathrm{s}$ with constant acceleration at the same time; in part $T 2$, pitch angle ranges from $0^{\circ}$ to $10^{\circ}$; meanwhile, the velocity of flight body changes from $5 \mathrm{~m} / \mathrm{s}$ to $10 \mathrm{~m} / \mathrm{s}$ with constant acceleration; in part $T 3$, roll angle ranges from $0^{\circ}$ to $5^{\circ}$, and the flight velocity also changes from $10 \mathrm{~m} / \mathrm{s}$ to $5 \mathrm{~m} / \mathrm{s}$ with constant acceleration. The attitude and velocity variation curves generated by trajectory generator are shown in Figure 7.

The constant drifting of the gyro $0.01^{\circ} / \mathrm{h}$, the constant bias of accelerometer $50 \mathrm{ug}$ and the angle sensor error $2^{\prime \prime}$ are added to the ideal navigation data generated by trajectory generator to produce semiphysical simulation data.

\subsubsection{The POS Performance Comparison Experiments When} Flight Body Moves with Variable Velocity. Reading the semiphysical simulation data added to sensor errors, the attitude of the imaging payloads is calculated by separated POS and centralized POS, respectively. The attitude errors are shown in Figure 8(a), and the minute differences between the attitude errors of two POSs are shown in Figure 8(b). According to Figure 8 , the attitude error characteristics can be displayed visually to analyze whether the equivalence of the separated and centralized POS exists or not when flight body moves with variable velocity.

From Figure 8, the attitude errors of the separated POS are similar to that of centralized POS. And the maximum three-axis attitude (azimuth angle, pitch angle, and roll angle) errors of separated POS/centralized POS, caused by sensor errors, are $5.968 \times 10^{-5^{\circ}} / 1.443 \times 10^{-4^{\circ}}, 2.946 \times 10^{-3^{\circ}} / 2.728 \times$ $10^{-3^{\circ}}$, and $5.395 \times 10^{-4^{\circ}} / 7.493 \times 10^{-4^{\circ}}$, respectively. The minute differences between attitude errors of separated POS 

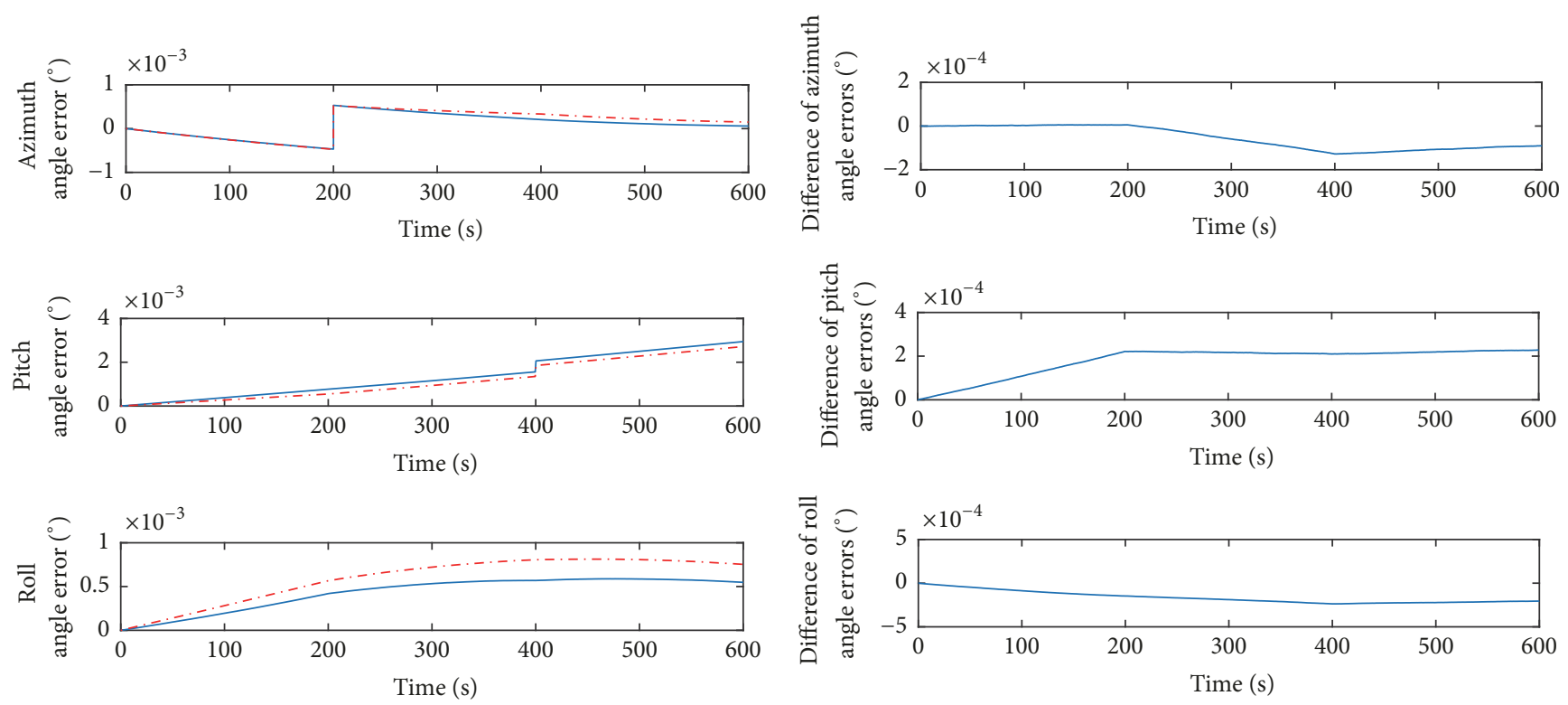

- Separated pos attitude error

-.- Centralized pos attitude error

(a) The attitude errors of separated POS and centralized POS compared with the ideal attitudes

(b) The differences between attitude errors of separated POS and centralized POS

FIGURE 8: The attitude errors with variable velocity motion.

and centralized POS during the whole process are shown in Figure $8(\mathrm{~b})$. And the maximum differences of the three-axis attitude errors are $1.272 \times 10^{-4^{\circ}}, 2.173 \times 10^{-4^{\circ}}$, and $2.098 \times$ $10^{-4^{\circ}}$, which can be also ignored in contrast to the attitude error shown in Figure 8(a).

Therefore, the attitude calculation results of the two POSs are similar; namely, the equivalence of the separated POS and the centralized POS is validated even if the flight body moves with variable velocity.

\section{Conclusion}

Based on the analysis for characteristics of remote sensing system, the design idea of separated POS integrated with ISP system is proposed in this paper. The attitude of imaging payloads is obtained after calculating the inertial parameters from three gyros and accelerometers mounted on different gimbals and effective information introduced by flight inertial navigation system under the principle of coordinate transformation. The separated POS not only simplifies the mechanical and electronic interfaces between imaging payloads and ISP system but also improves the transmission precision of angular velocities among gimbals. Moreover, the signals provided by gyros can also be applied as rate feedback directly which decreases the number of inertial sensors used in the ISP system but improves the control precision of gimbals. A series of theoretical derivations and semiphysical simulations under different motion conditions validate the correctness and effectiveness of design scheme presented in this paper. Besides the effective function equal to centralized system, the separated POS enhances the integration with the ISP system and decreases the cost and volume of remote sensing system. Furthermore, it improves the design independence of the imaging payloads significantly which predicts the good application prospects.

\section{Conflicts of Interest}

The authors declare that there are no conflicts of interest regarding the publication of this paper.

\section{Acknowledgments}

This work is supported by the National Natural Science Foundation of China under Grant no. 61473019, Beijing Natural Science Foundation under Grant no. 4172036, Beijing Science and Technology Plan under Grant no. D171100006217003, and Open Research Fund of the State Key Laboratory of Tianjin Key Laboratory of Intelligent Information Processing in Remote Sensing under Grant no. 2016-ZW-KFJJ-01.

\section{References}

[1] X.-L. Chen, H.-M. Zhao, P.-X. Li, and Z.-Y. Yin, "Remote sensing image-based analysis of the relationship between urban heat island and land use/cover changes," Remote Sensing of Environment, vol. 104, no. 2, pp. 133-146, 2006.

[2] S. Gairola, Ş. Procheş, and D. Rocchini, "High-resolution satellite remote sensing: A new frontier for biodiversity exploration in Indian Himalayan forests," International Journal of Remote Sensing, vol. 34, no. 6, pp. 2006-2022, 2013. 
[3] M. Meroni, A. Schucknecht, D. Fasbender et al., "Remote sensing monitoring of land restoration interventions in semi-arid environments with a before-after control-impact statistical design," International Journal of Applied Earth Observation and Geoinformation, vol. 59, pp. 42-52, 2017.

[4] K. Xu, X. Zhang, Z. Chen, W. Wu, and T. Li, "Risk assessment for wildfire occurrence in high-voltage power line corridors by using remote-sensing techniques: a case study in Hubei Province, China," International Journal of Remote Sensing, vol. 37, no. 20, pp. 4818-4837, 2016.

[5] N. Thomas, C. Hendrix, and R. G. Congalton, "A comparison of urban mapping methods using high-resolution digital imagery," Photogrammetric Engineering and Remote Sensing, vol. 69, no. 9, pp. 963-972, 2003.

[6] Y. Zou and X. Lei, "A compound control method based on the adaptive neural network and sliding mode control for inertial stable platform," Neurocomputing, vol. 155, pp. 286-294, 2015.

[7] X. Zhou, G. Gong, J. Li, H. Zhang, and R. Yu, "Decoupling control for a three-axis inertially stabilized platform used for aerial remote sensing," Transactions of the Institute of Measurement and Control, vol. 37, no. 9, pp. 1135-1145, 2015.

[8] Y. Zhang, C. Du, and Q. Mu, "Random error modelling and compensation of accelerometer in airborne remote sensing stabilized platform," Transactions of the Institute of Measurement and Control, vol. 35, no. 4, pp. 503-509, 2013.

[9] J. M. Hilkert, "Inertially stabilized platform technology: concepts and principles," IEEE Control Systems Magazine, vol. 28, no. 1, pp. 26-46, 2008.

[10] M. K. Masten, "Inertially stabilized platforms for optical imaging systems: tracking dynamic targets with mobile sensors," IEEE Control Systems Magazine, vol. 28, no. 1, pp. 47-64, 2008.

[11] H. G. Wang and T. C. Williams, "Strategic inertial navigation systems: high-accuracy inertially stabilized platforms for hostile environments," IEEE Control Systems Magazine, vol. 28, no. 1, pp. 65-85, 2008.

[12] Q. Mu, G. Liu, and X. Lei, "A RBFNN-Based Adaptive Disturbance Compensation Approach Applied to Magnetic Suspension Inertially Stabilized Platform," Mathematical Problems in Engineering, vol. 2014, Article ID 657985, 2014.

[13] X. Zhou, Y. Jia, Q. Zhao, and T. Cai, "Dual-rate-loop control based on disturbance observer of angular acceleration for a three-axis aerial inertially stabilized platform," ISA Transactions $^{\circledR}$, vol. 63, pp. 288-298, 2016.

[14] J. Fang and R. Yin, "An adaptive nonlinear control for gyro stabilized platform based on neural networks and disturbance observer," Mathematical Problems in Engineering, vol. 2014, Article ID 472815, 2014.

[15] X. Zhou, B. Zhao, W. Liu, H. Yue, R. Yu, and Y. Zhao, "A compound scheme on parameters identification and adaptive compensation of nonlinear friction disturbance for the aerial inertially stabilized platform," ISA Transactions ${ }^{\circledR}$, vol. 67, pp. 293305, 2017.

[16] J. C. Fang, Z. H. Qi, and M. Y. Zhong, "Feedforward compensation method for three axes inertially stabilized platform imbalance torque," Journal of Chinese Inertial Technology, vol. 18, no. 1, pp. 38-43, 2010.

[17] C. Bai and Z. Zhang, "Acceleration-based mass imbalance feedforward compensation for inertial stabilized platform," International Journal of Control, Automation, and Systems, vol. 12, no. 3, pp. 609-617, 2014.
[18] S. Li and M. Zhong, "High-precision disturbance compensation for a three-axis gyro-stabilized camera mount," IEEE/ASME Transactions on Mechatronics, vol. 20, no. 6, pp. 3135-3147, 2015.

[19] Y. Zhang and R. Zhu, "A novel gyro installation mode of threeaxis stabilized platform used for airborne earth observation," Hangkong Xuebao/Acta Aeronautica et Astronautica Sinica, vol. 31, no. 3, pp. 614-619, 2010.

[20] W. Ji, Q. Li, B. Xu, D. Zhao, and S. Fang, "Adaptive fuzzy PID composite control with hysteresis-band switching for line of sight stabilization servo system," Aerospace Science and Technology, vol. 15, no. 1, pp. 25-32, 2011. 


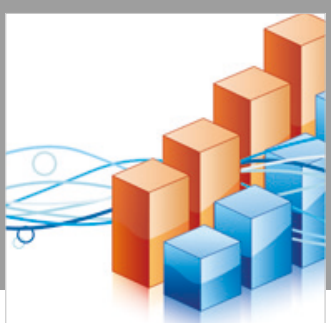

Advances in

Operations Research

\section{-n-m}
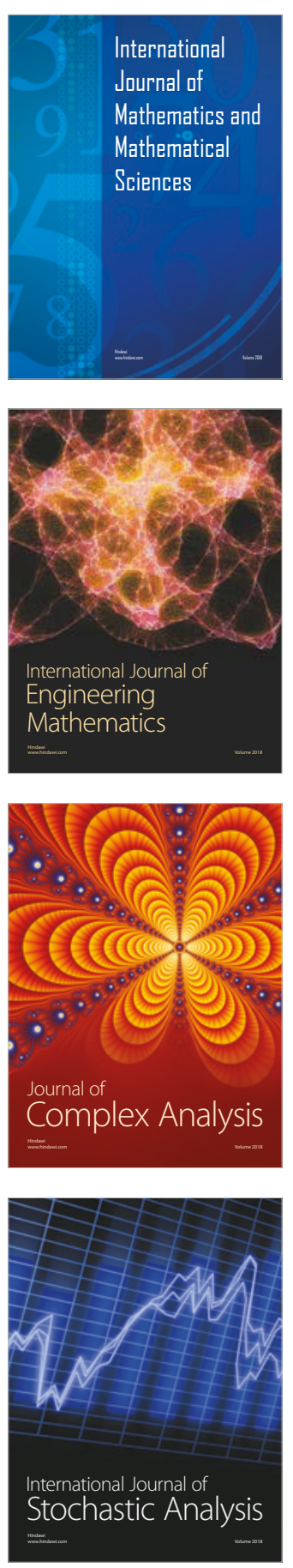
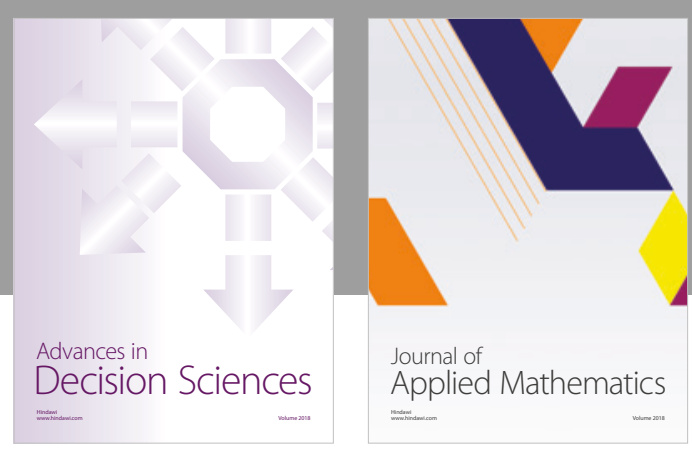

Journal of

Applied Mathematics
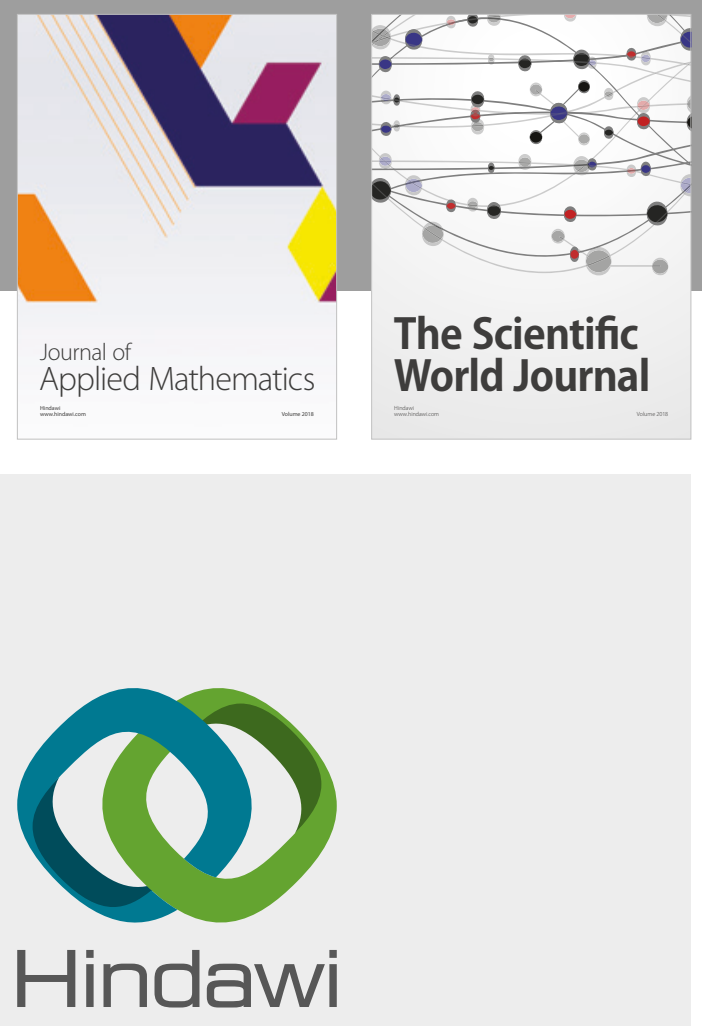

Submit your manuscripts at

www.hindawi.com

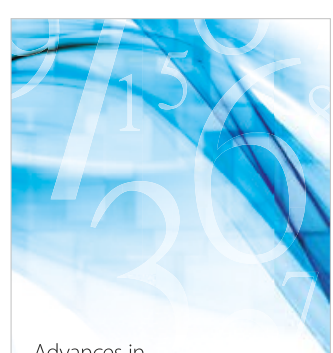

Advances in
Numerical Analysis
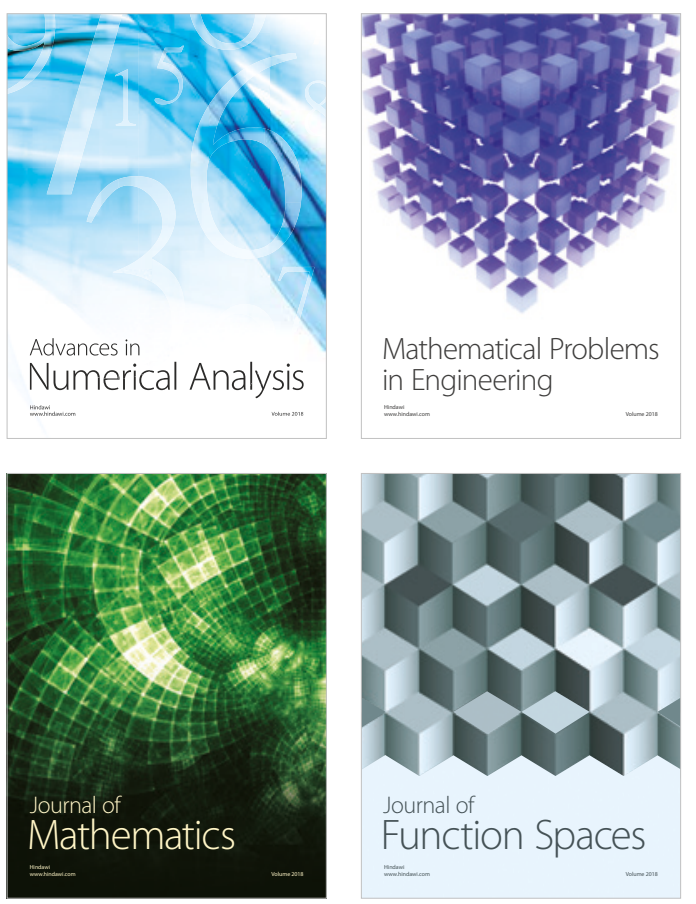

Mathematical Problems in Engineering

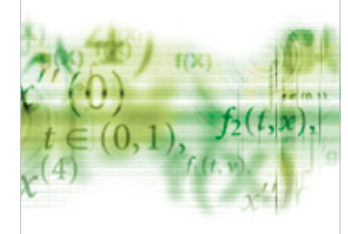

International Journal of

Differential Equations

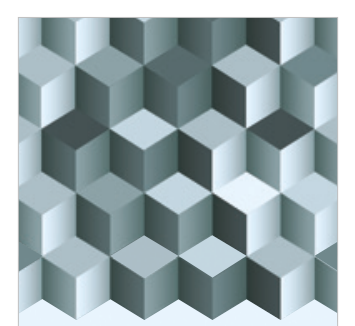

Journal of

Function Spaces

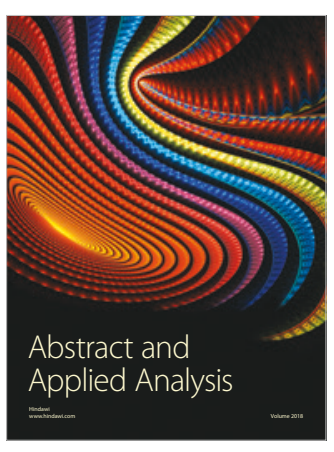

The Scientific

World Journal

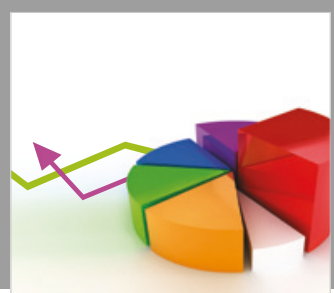

Journal of

Probability and Statistics
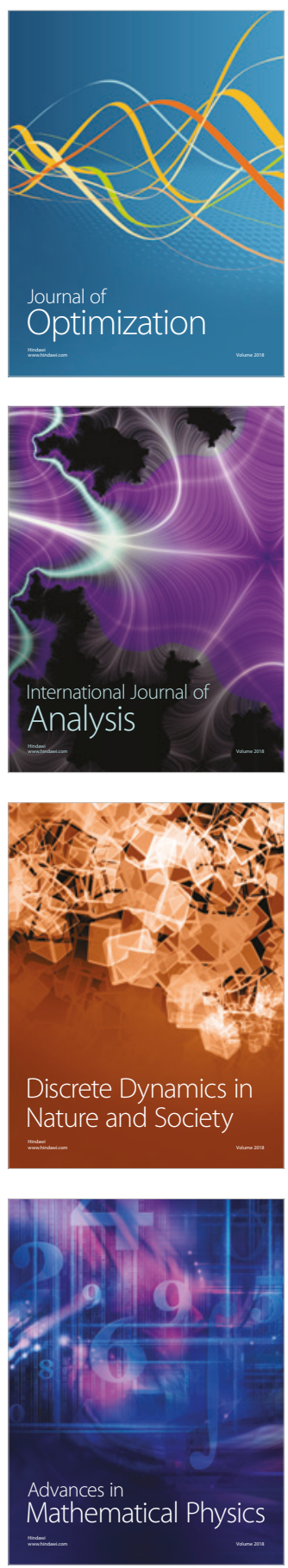